\title{
SMILA: A COMPACT AND EFFICIENT ANTENNA FOR MOBILE COMMUNICATIONS
}

\section{J.-F. Zürcher, ${ }^{1}$ O. Staub, ${ }^{1}$ and A. K. Skrivervik ${ }^{1}$ \\ ${ }^{1}$ Laboratoire d'Electromagnétisme et d'Acoustique Ecole Polytechnique Fédérale de Lausanne \\ $\mathrm{CH}-1015$ Lausanne, Switzerland}

\section{Received 15 May 2000}

ABSTRACT: A new antenna, integrated with the shielding structure of the device, is described. Despite its compact dimensions, it has excellent radiation properties. (c) 2000 John Wiley \& Sons, Inc. Microwave Opt Technol Lett 27: 155-157, 2000.

Key words: electrically small antennas; mobile communications; PCS; DECT; GSM; PIFA; SMILA

\section{INTRODUCTION}

With the development of miniaturized devices for mobile communications, the need for compact and efficient antennas increases. Ideally, these antennas should be simple and extremely inexpensive to manufacture, while having good electrical performances, and especially a high efficiency. This is important because, due to limited battery space, the dc power is always limited, a fact which limits the RF power even more. On the receiver side, any additional decibels of gain provided by the antenna improve the $S / N$ ratio at no battery consumption cost.

Typical applications for such antennas are DECT or GSM.

\section{ANTENNA DEVELOPMENT}

The application for which this antenna has been developed is in the 1.8-1.9 GHz band (DECT/DCS), and the mechanical constraints were stringent: a metallic cylindrical case measuring $35 \mathrm{~mm}$ in diameter with a height of $10 \mathrm{~mm}$.

To optimize the performances of an electrically small antenna, it must fill most of the available volume, while losses should be as low as possible. One way to attain this goal is to locate the radiating part around the outer wall of the cylindrical box to maximize the antenna dimensions.

The development of the following design started from the PIFA (planar inverted- $F$ antenna) [1], which has following advantages:

- low losses (no dielectric, thick metal)

- simple concept

- easy to match (position of the feed)

- easy to tune (adjusting the length of the arm).

A classical PIFA is depicted in Figure 1. The properties of this antenna can be adjusted when varying the various relative dimensions:

- an increase in $H$ widens the bandwidth

- reducing $W(W<L 1)$ reduces the overall dimensions, but also the bandwitch

- L2 allows a frequency tuning

- every modification changes the position of the feedpoint for a given impedance.

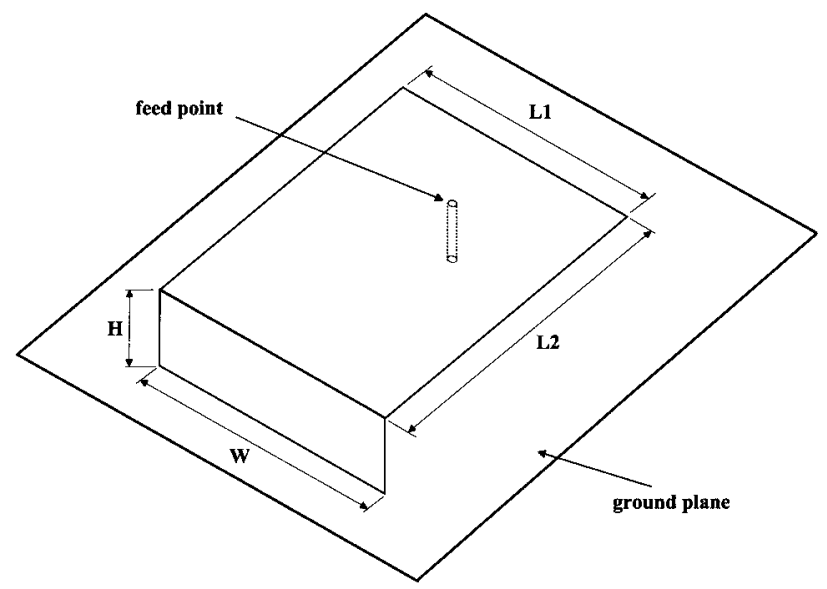

Figure 1 Standard PIFA with its main dimensions

In its most usual shape, the PIFA has approximately the proportions shown in Figure 1. For our application, the shape will be closer to a strip ( $W \ll L 2$ ), and the width of the short circuit will be the same as the antenna width $(W=L 1)$. Furthermore, the antenna height $H$ will be small due to the limited space. For such an antenna, the feedpoint has to be placed close to the short-circuited end to obtain a $50 \Omega$ impedance, and the bandwidth will be reduced due to the reduction in dimensions and height. On the other hand, it has been shown that the bandwidth of a PIFA increases when the ground plane is reduced [1].

The study started with the design and test of a narrow PIFA ( $W=L 1=10 \mathrm{~mm}$, similar to the case height) having a height $H$ between 3 and $4 \mathrm{~mm}$. Simulations have been made with HP ADS/Momentum, which gave a fairly good match with measured results. On the realized prototypes, the ground plane, which was initially $80 \times 80 \mathrm{~mm}$ large, was progressively reduced. The effect on the resonant frequency was negligible, but the $-10 \mathrm{~dB}$ bandwidth increased. This is consistent with data published in the literature [1].

Then, a bent PIFA mounted on the side of a round box was simulated. The simulation could no longer be made with HP ADS/Momentum since the geometry is not planar. Tentative attempts have been made to simulate this structure with HP HFSS; however, the simulation times were excessive, even with a reduced number of tetrahedrons. Other tests have been made using NEC, which gave acceptable results.

A prototype was constructed and tested. The frequency could very easily be tuned by cutting the length of the radiating strip, while the matching could be adjusted by varying the position of the feed point relative to the short-circuited side. This position is relatively critical (a few tenths of a millimeter).

Finally, the antenna was fully integrated within the box. Figure 2 shows the evolution from the "classical" PIFA to the version integrated with the box.

This version has been called SMILA (smart monoblockintegrated L-antenna), and is depicted more in detail in Figure 3.

The final prototype has been drawn with the CAD program SolidWorks, and machined by $\mathrm{CNC}$ out of a massive brass block. 


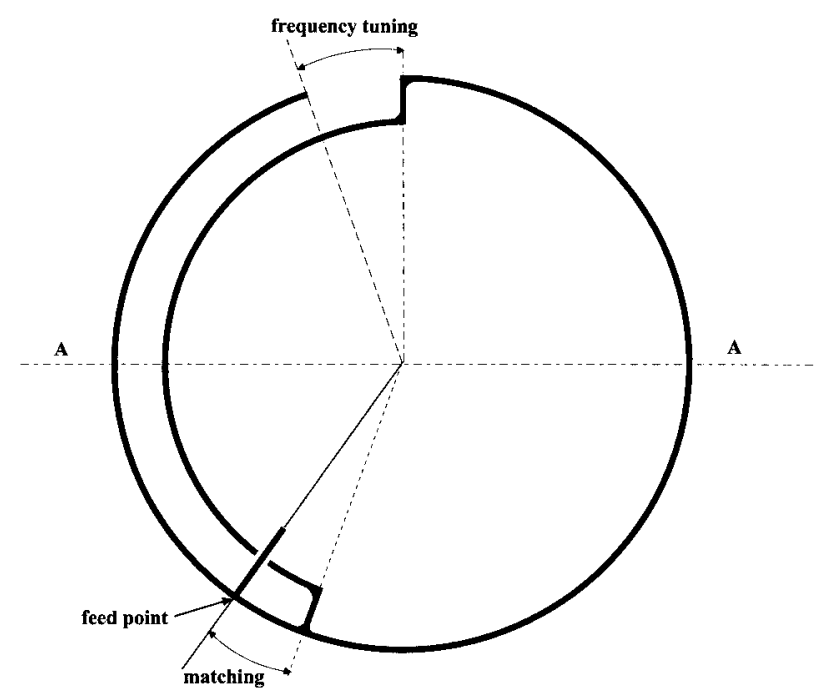

b) bending the PIFA around the metallic box

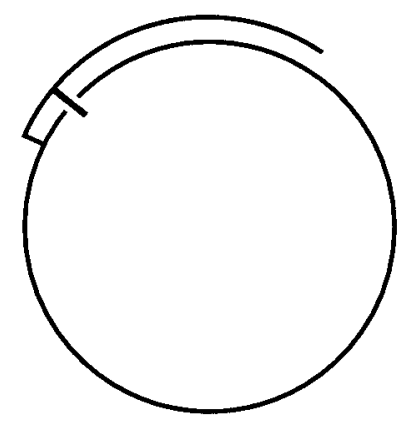

\section{c) integrating the PIFA within the metallic box}

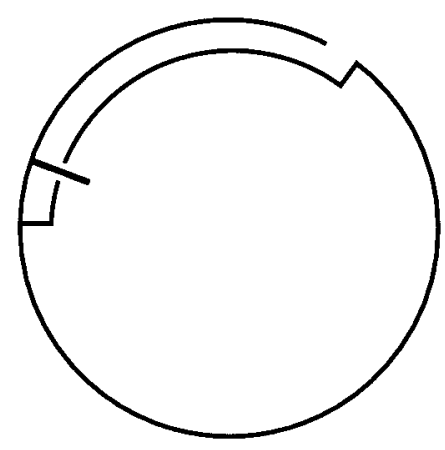

Figure 2 From PIFA to SMILA

\section{MEASURED PERFORMANCES}

The performances of the SMILA have been measured accurately. Figure 4 shows the measured SWR. The bandwidth for an SWR $<2$ is $77.3 \mathrm{MHz}$, corresponding to $4.1 \%$. It must be noted that the $S_{11}$-measurement using a network analyzer is influenced by surface waves propagating around the cable. This is due to the fact that the antenna is very small (about $\lambda / 5$ ). To make proper measurements, absorbing material had to be put around the cable.

To measure the maximum gain and radiation patterns, any connection to the outside world should be avoided as this would completely modify the results [2]. The technique described in [3] has been used: a VCO and a miniature battery have been integrated into the SMILA box. Figure 5 shows the prototype used for measurements.

The measured peak gain is $+0.8 \mathrm{dBi}$, very close to the theoretical limit [4], which is $2.2 \mathrm{dBi}$ (for a perfect antenna completely filling a sphere having the diameter of our cylindrical case). This good result is due to the nature of the antenna: no dielectric, relatively thick metallic parts $(0.5 \mathrm{~mm}$ thickness), and is also due to the fact that the antenna size

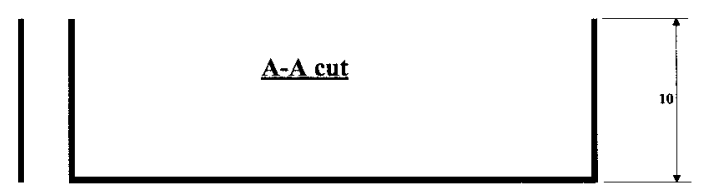

Figure 3 Detailed view of the SMILA showing the important parameters

occupies a maximum of volume, and that the case itself participates in the radiation.

The radiation patterns have been measured, and show no deep null: the patterns are relatively omnidirectional, as can be expected from an electrically small antenna.

It has to be noted that, for such a small antenna, the polarization is not well defined. This is not a drawback since, in mobile communications applications, there are always many reflections which randomly degrade the polarization purity.

One limitation of such an antenna is the lower frequency limit as a function of the box dimensions. For instance, for a $35 \mathrm{~mm}$ diameter box, the lower limit is about $800 \mathrm{MHz}$ : at this frequency, the antenna would occupy the entire circumference of the box. Another limitation is the bandwidth: it could be increased by increasing the antenna height $H$, but this is not always possible due to design considerations. But these limitations are common to all antennas which are small with respect to the wavelength.

\section{CONCLUSIONS}

The SMILA described here is a simple and efficient antenna, which can easily be manufactured as an integral part of the metallic box enclosing the electronic, reducing the costs to practically zero. Its frequency and impedance tuning are very simple. In the described application, its shape is round, but any other shape can be envisaged, depending on the application. To the authors' knowledge, it has the maximum possible peak gain within the available volume, and its bandwidth is sufficient for many applications.

This antenna has been patented (Swiss Patent 1999 1849/99 and European Patent 99120230.0) 

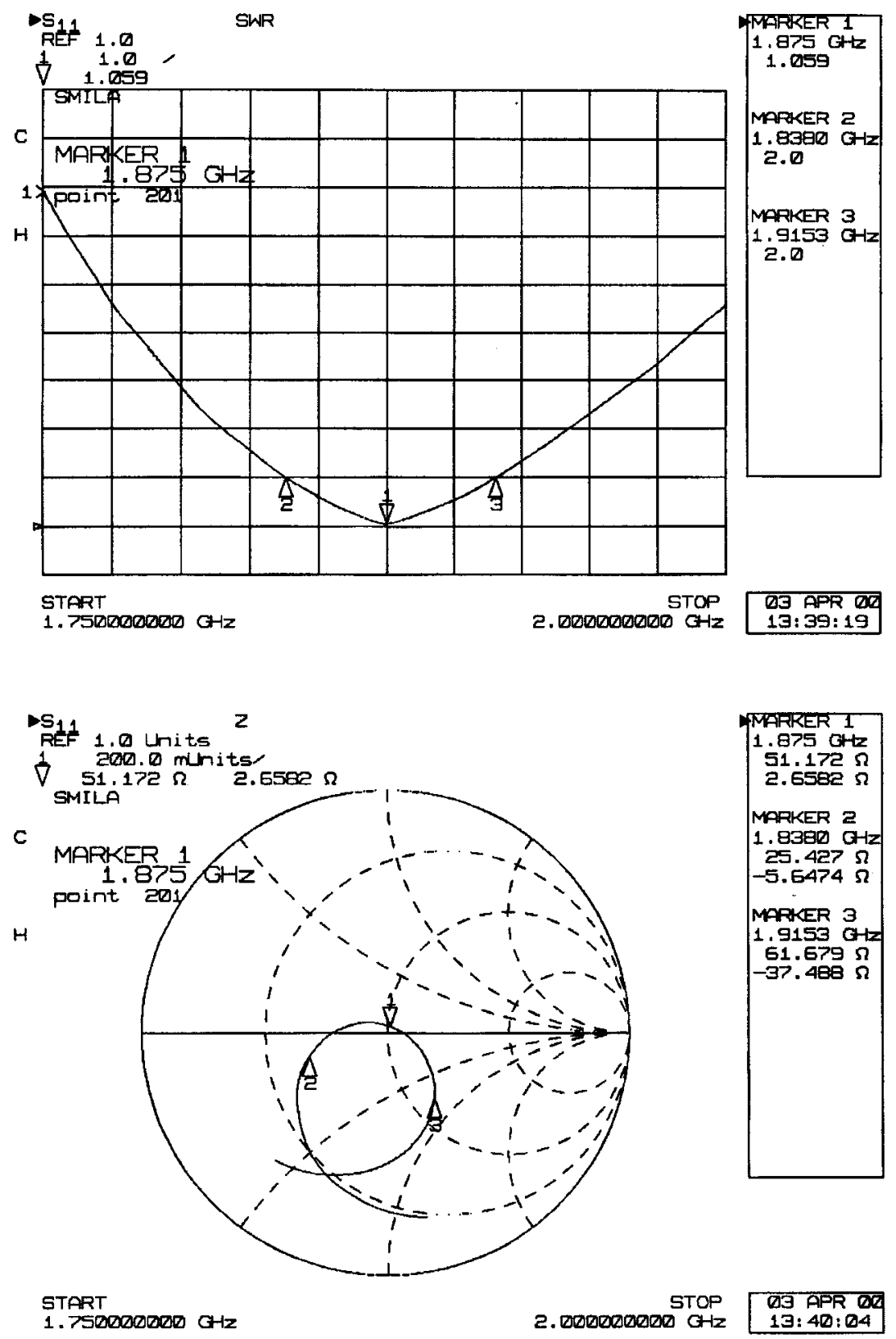

Figure 4 Measured SWR and Smith chart of the SMILA

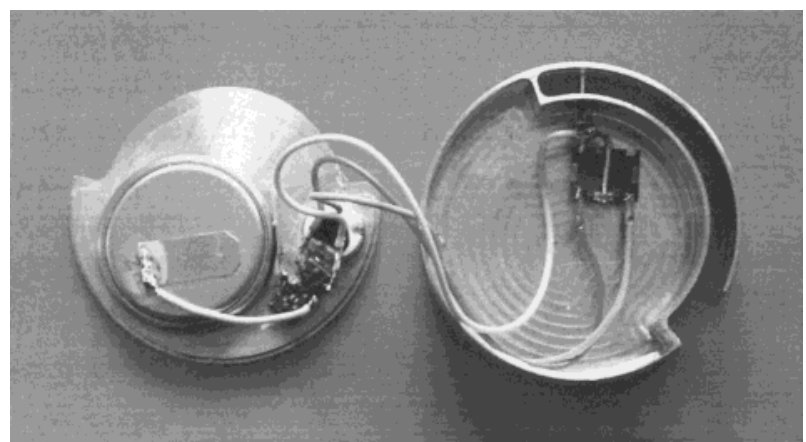

Figure 5 SMILA prototype used for the measurements, with integrated VCO and batteries

\section{REFERENCES}

1. T. Taga, "Analysis of planar inverted-F antennas and antenna design for portable radio equipment," Analysis, design and measurement of small and low-profile antennas, K. Hirasawa and M. Haneishi (Editors), Artech House, Norwood, MA, 1992, pp. 161-180.

2. O. Staub, J.-F. Zürcher, A. Skrivervik, Some considerations on the correct measurement of the gain and bandwidth of electrically small antennas, Microwave Opt Technol Lett 17 (1998), 156-160.

3. J.-F. Zürcher, O. Staub, A.K. Skrivervik, and M. Hermanjat, Accurate measurement of the maximum gain of electrically small antennas, Microwave Opt Technol Lett 23 (1999), 328-331.

4. R.F. Harrington, Effect of antenna size on gain, bandwidth and efficiency, J Res Nat Bureau Standards-D, Radio Propagat 64D (1960), 1-12.

(c) 2000 John Wiley \& Sons, Inc. 